\title{
ALIO/EURO V CONFERENCE ON COMBINATORIAL OPTIMIZATION (ENST, PARIS, 26-28 OCTOBER 2005)
}

The Association of Latin-Iberoamerican Operational Research Societies (ALIO; see http://www-2.dc.uba.ar/alio/) and the Association of European Operational Research Societies (EURO; see http://www.euro-online.org/), both within the International Federation of Operational Research Societies (IFORS; see http://www.ifors.org/), organize a joint conference every third year, devoted to Combinatorial Optimization. The main purpose of the ALIO/EURO Conferences on Combinatorial Optimization is to bring together researchers from different countries, especially Latin American and European, and to stimulate activities and discussions about methods and applications in the field of combinatorial optimization.

After Brazil (1989), Chile (1996), Italy (1999), and Chile (2002), the Fifth ALIO/EURO Conference on Combinatorial Optimization was held in Paris (France), at the École nationale supérieure des télécommunications (ENST), on October 26-28, 2005 (see http://www.infres.enst.fr/ ${ }^{\sim}$ charon/alio/ for more details).

About 100 talks were selected for this conference. They were gathered into sessions reflecting the methods or the applications arising in combinatorial optimization: Integer programming, Quadratic programming, Local and global search, Metaheuristics, Relaxations, Polyhedral methods, Branching methods, Dynamic programming, Algorithms, Graph algorithms, Graphs, Trees, Networks, Coloration problems, Timetables, Scheduling, Assignment problems, Vehicle routing problems, Ordering problems, Clustering, Partitioning, Lot sizing, Decision aid, Multicriteria and multi-objective approaches, Statistics, Bio-informatics. More than 150 participants attended the conference, coming from about 30 countries: Algeria, Argentina, Austria, Belgium, Brazil, Canada, Chile, Colombia, Czech Republic, Denmark, Finland, France, Germany, Iran, Italy, Luxembourg, Mexico, the Netherlands, Norway, Portugal, Russia, Spain, Switzerland, Ukraine, United Kingdom, the United States of America, Venezuela...

After the conference, 19 full papers were submitted for publication in the special issue of RAIRO-Operations Research devoted to the ALIO/EURO V Conference on Combinatorial Optimization. The usual high standards of the journal were applied through a strict, thorough, and selective refereeing process. Then, this 
refereeing process leads us to select the following five papers to be published in this special issue:

- C. Alves, J.M. Valerio de Carvalho, A branch-and-price-and-cut algorithm for the pattern minimization problem. The pattern minimization problem consists, in cutting stocks problems, in minimizing the number of setups. An exact method is designed to solve this NP-hard problem, based on column generation, on branch and bound, and on cutting planes.

- R. Figueiredo, V. Barbosa, N. Maculan, C. de Souza, Acyclic orientations with path constraints. The authors design a linear programming formulation of acyclic orientations with path constraints. They then discuss its application in solving the vertex colouring problem and some versions of the frequency assignment problem.

- Alain Guénoche, Comparison of algorithms in graph partitioning. This paper describes five methods to cluster vertices of a graph. These methods are then compared according to their abilities to recover classes initially introduced in random graphs with more edges inside classes than between them.

- Arie M. C. A. Koster, Annegret K. Wagler, Comparing imperfection ratio and imperfection index for graph classes. It is well-known that, for any graph $G$, the stable set polytope $\operatorname{STAB}(G)$ of $G$ is included into the fractional stable set polytope $\operatorname{QSTAB}(G)$ of $G$, with an equality for perfect graphs. This paper investigates three different concepts about these polytopes, involving the facet set of $\operatorname{STAB}(G)$, the disjunctive index of $\operatorname{QSTAB}(G)$, and the dilation ratio of these two polytopes.

- S. Varone, N. Zufferey, Three tabu search methods for the MI-FAP applied to 802.11 networks. In this paper, the authors consider the minimum interference frequency assignment problem (MI-FAP), which is NP-hard. They design three tabu search heuristics and then compare them, based on time constraints and on the quality of their solutions.

We seize this opportunity to thank the RAIRO-Operations Research journal for publishing these articles, as well as its editors-in-chief, Alain Billionnet, Philippe Chrétienne and Philippe Mahey, for their advices and their help in the publication of this special issue. We would like also to thank the anonymous referees, the scientific committee of the conference and our institution, the ENST. The next

ALIO/EURO Conference will be located in Buenos Aires, Argentina, on December $15-17,2008$.

IRÈnE CHARON

OLIVIER HUdRY 